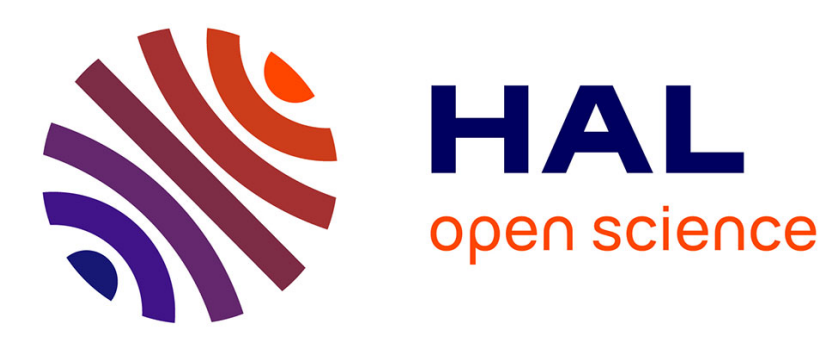

\title{
Pinning Stabilizes Neighboring Surface Nanobubbles against Ostwald Ripening
}

\author{
Benjamin Dollet, Detlef Lohse
}

\section{To cite this version:}

Benjamin Dollet, Detlef Lohse. Pinning Stabilizes Neighboring Surface Nanobubbles against Ostwald

Ripening. Langmuir, 2016, 32 (43), pp.11335 - 11339. 10.1021/acs.langmuir.6b02136 . hal-01391231

\section{HAL Id: hal-01391231 \\ https://hal.science/hal-01391231}

Submitted on 7 Nov 2016

HAL is a multi-disciplinary open access archive for the deposit and dissemination of scientific research documents, whether they are published or not. The documents may come from teaching and research institutions in France or abroad, or from public or private research centers.
L'archive ouverte pluridisciplinaire HAL, est destinée au dépôt et à la diffusion de documents scientifiques de niveau recherche, publiés ou non, émanant des établissements d'enseignement et de recherche français ou étrangers, des laboratoires publics ou privés. 


\title{
Pinning stabilizes neighboring surface nanobubbles against Ostwald ripening
}

\author{
Benjamin Dollet ${ }^{\dagger}$ and Detlef Lohse, \\ $\dagger$ Institut de Physique de Rennes (UMR CNRS 6251), Université Rennes 1, Rennes, France \\ $\ddagger$ Physics of Fluids Group, Department of Applied Physics, MESA+Research Center, and \\ J. M. Burgers Center for Fluid Dynamics, University of Twente, P.O. Box 217, 7500 AE \\ Enschede, The Netherlands \\ E-mail: d.lohse@utwente.nl
}

\begin{abstract}
Pinning of the contact line and gas oversaturation explain the stability of single surface nanobubbles. In this article we theoretically show that the pinning also suppresses the Ostwald ripening process between neighboring surface nanobubbles, thus explaining why in a population of neighboring surface nanobubbles different radii of curvature of the nanobubbles can be observed.
\end{abstract}

\section{Introduction}

When a solid is immersed in a gas-oversaturated liquid, stable nanoscopic spherical-cap shaped so-called surface nanobubbles can be formed at the interface. ${ }^{1-5}$ These were first speculated to exist based on stepwise features in atomic forces curves between hydrophobic surfaces ${ }^{6}\left[\frac{8}{6}\right.$ and later seen in atomic force microscopy. ${ }^{[11}$ Meanwhile it has been theoretically understood and supported by numerical simulations that the reason for the on first sight surprising stability of a single surface nanobubbles lies in the balance between gas overpressure thanks to the gas oversaturation in the liquid and the Laplace pressure $\mathrm{e}^{112} \frac{14}{14}$ and that pinning of the contact line $\mathrm{I}^{\sqrt{15}-18}$ is a necessary condition to achieve this stability for a single surface nanobubble. 
What however happens when there are several surface nanobubbles sitting next to each other on a surface? On one hand the theory of Lohse and Zhang $\frac{13}{13}$ predicts that for given gas oversaturation $\zeta=c_{\infty} / c_{s}-1>0$ the equilibrium contact angle $\theta_{e q}$ of all these surface bubbles should obey

$$
\sin \theta_{e q}=\zeta \frac{L}{L_{c}}
$$

where $L$ is the footprint diameter of the pinned bubble (assumed to be of spherical cap-shape) and $L_{c}=4 \sigma / P_{0}$ following from the surface tension $\sigma$ and the ambient pressure $P_{0}$. Moreover, $c_{s}$ is the saturation concentration and $c_{\infty}$ is the given gas concentration far away from the bubble. Eq. (1) implies that the equilibrium radius of curvature $R_{e q}=L /\left(2 \sin \theta_{e q}\right)$ should be the same for all surface nanobubbles, namely

$$
R_{e q}=\frac{L_{c}}{2 \zeta_{e q}} .
$$

On the other hand one would expect that neighboring surface nanobubbles are subjected to Ostwald ripening, ${ }^{19}$ leading to shrinkage of bubbles with small radius of curvature and growth of neighboring ones with large radius of curvature. Indeed, experimental studies do reveal neighboring surface nanobubbles with different radii of curvature. $\frac{15520 \mid 26}{26}$

The solution to this apparent paradox has qualitatively been given in the last paragraph of the seminal paper by Zhang and coworker, $\frac{15}{15}$ who not only identified contact line pinning as reason for the stabilization of single surface nanobubbles against dissolution, but also as reason for the stabilization against Ostwald ripening within a population of surface nanobubbles. The aim of this paper is to theoretically and quantitatively work out this idea. We will study the stability of two different neighboring bubbles with distance $d$ against Ostwald ripening, where $d$ is assumed to be much larger than the footprint diameter of the bubbles. The problem is mathematically formulated in the next section. Then the results of the linear stability analysis are presented and discussed. The paper closes with conclusions and an outlook. 


\section{Derivation of the evolution equation for two diffusively cou- pled surface bubbes}

We state the problem as follows: consider two pinned surface nanobubbles, labelled 1 and 2, of constant footprint lengths $L_{1}$ and $L_{2}$ and variable contact angle $\theta_{1}$ and $\theta_{2}$, separated by a distance $d$ (Fig. 1). Let $c(\mathbf{x}, t)$ be the concentration field of dissolved gas in the liquid surrounding the nanobubbles. The boundary conditions are: $c \rightarrow c_{\infty}$ far from the nanobubbles, $\partial c / \partial n=0$ at the solid boundary (i.e., no flux through it), where $n$ is the normal direction to the boundary, and Henry's law at $S_{i}$, the surface of nanobubble $i: 1$

$$
\left.c\right|_{S_{i}}=c_{s}\left(1+\frac{4 \sigma \sin \theta_{i}}{L_{i} P_{0}}\right)
$$

where $\sigma$ is the surface tension, and $P_{0}$ the ambient pressure. In the absence of bulk flow, the concentration field obeys the diffusion equation: $\partial c / \partial t=D \Delta c$, with $D$ the diffusion coefficient of the dissolved gas. We will further assume that the evolution of the nanobubbles is slow enough to be considered as quasistatic for the diffusion problem, so that the concentration field simply obeys the Laplace equation:

$$
\Delta c=0
$$

This quasistatic approximation does not rule out the possibility of Ostwald ripening, ${ }^{19}$ provided that the time scale of this process is much larger than the diffusive time scale $\tau_{D}=L^{2} / D$, where the footprint $L$ is taken as a relevant characteristic length scale of the nanobubble. Actually, the quasistatic approximation is well suited for nanobubbles (say, $L<1 \mu \mathrm{m}$ ), for which $\tau_{D}$ is below $1 \mathrm{~ms}$, using $D \approx 10^{-9} \mathrm{~m}^{2} / \mathrm{s}$ as the typical diffusion coefficient of gases in water. ${ }^{27}$

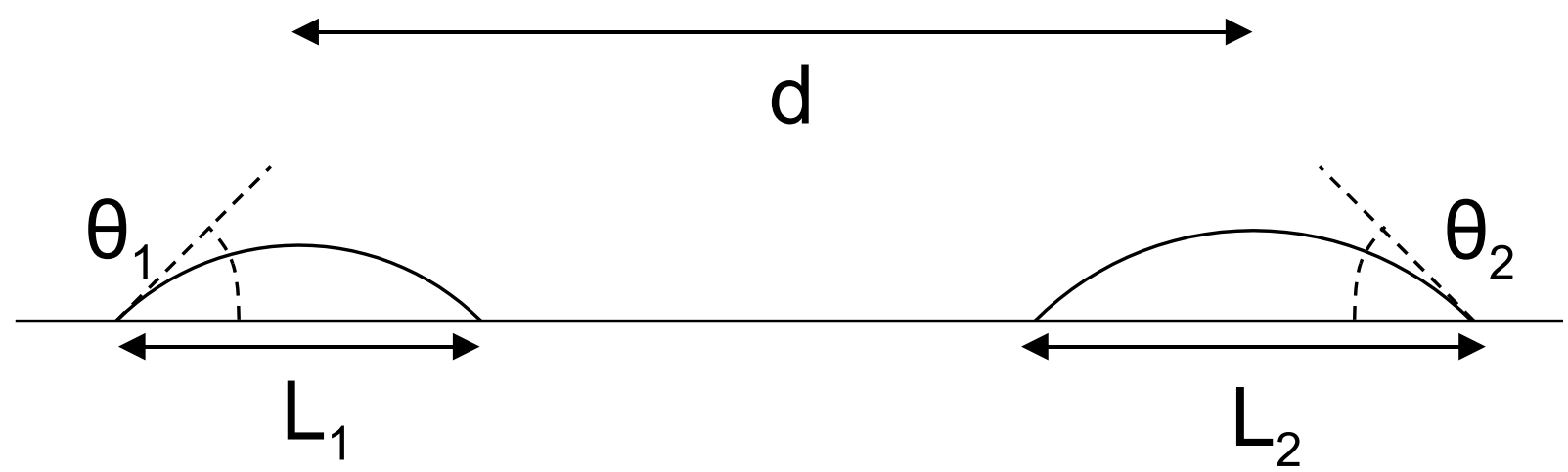

Figure 1: Sketch of the two nanobubbles. 
In general, this problem must be solved numerically. However, like for other interaction problems involving potential flows, such as the relative translation of two spheres ${ }^{28}$ or the coupled dynamics of two bubbles in an acoustic field, $\stackrel{29}{2}$ we can resort to analytical approximations when the nanobubbles are far enough from each other so that $L_{i} / d \ll 1$. Formally, we introduce the small parameter

$$
\varepsilon=\frac{L_{1}}{d}
$$

and we assume that $L_{2} / L_{1}$ is of order 1 , so that $L_{2} / d$ is of the same order $\varepsilon$. In this case, the potential field (here, the concentration field) can be expanded in a power series in $\varepsilon$, which dominant terms represent single objects without interaction, and with correction terms accounting for the interaction. A way to construct such a series is to consider successive images of a sphere with respect to the other. ${ }^{28}$ However, we cannot use such an approach in our case, except for the very special case $\theta_{1}=\theta_{2}=\pi / 2$. Actually, this case can be treated exactly using bispherical coordinates.

We will use a rougher approximation, but fully consistent as a leading-order correction. Since the Laplace equation (4) is linear, we can use the principle of superposition of solutions. We split the concentration field as follows, where the constant term $c_{\infty}$ is substracted off for convenience:

$$
c=c_{\infty}+c_{1}^{(0)}+c_{2}^{(0)}+\varepsilon\left(c_{1}^{(1)}+c_{2}^{(1)}\right)
$$

where each subfield $c_{i}^{(j)}$ is of order 1 , and obeys Laplace equation: $\Delta c_{i}^{(j)}=0$, with the following boundary conditions:

$$
c_{i}^{(j)}=0 \text { at } \infty, \quad \frac{\partial c_{i}^{(j)}}{\partial n}=0 \text { at the solid boundary }
$$

for the bubbles $i=1,2$ and the orders $j=0,1$. Hence, the concentration field defined by (6) obeys the boundary conditions at the wall and at infinity. At $S_{i}$, we specify the following boundary condition:

$$
c_{i}^{(0)}=c_{s}\left(1+\frac{4 \sigma \sin \theta_{i}}{L_{i} P_{0}}\right)-c_{\infty} .
$$

We do not specify any boundary condition for $c_{i}^{(0)}$ at $S_{3-i}$. Hence, the problems labelled with $j=0$ neglect interaction: $c_{\infty}+c_{i}^{(0)}$ is exactly the concentration of the nanobubble $i$ if it 
were isolated. As a result, the fields $c_{i}^{(0)}$ take a nonzero value at $S_{3-i}$. Therefore, the field $c^{(0)}=c_{\infty}+c_{1}^{(0)}+c_{2}^{(0)}$ does not respect the boundary conditions 3 . This discrepancy is of order $\varepsilon$. Hence, the fields $c_{i}^{(1)}$ are introduced to compensate this discrepancy at order $\varepsilon$, by imposing as boundary condition over $S_{i}$ :

$$
c_{i}^{(1)}=-\left.\varepsilon^{-1} c_{3-i}^{(0)}\right|_{d},
$$

where $\left.c_{3-i}^{(0)}\right|_{d}$ is the concentration field induced by the presence of nanobubble $3-i$ evaluated at the location of the other nanobubble $i$. At order $\varepsilon$, it suffices to evaluate this term at a constant distance $d$; accounting for the spatial variations of $c_{3-i}^{(0)}$ along $S_{i}$ in 8 leads to terms of order $\varepsilon^{2}$, which we neglect. Here again, we do not specify boundary conditions for $c_{i}^{(1)}$ at $S_{3-i}$. Hence, as before, the field (6) does not respect the boundary condition (3), but the discrepancy is now of order $\varepsilon^{2}$. Hence, (6) gives the correct concentration field up to order $\varepsilon$ included.

To compute the evolution equation of nanobubble $i$, we need the diffusive mass flux $\dot{M}_{i}$ at its surface $S_{i}$. Consistently with (6), we write $\dot{M}_{i}=\dot{M}_{i}^{(0)}+\varepsilon \dot{M}_{i}^{(1)}$, where $\dot{M}_{i}^{(j)}=\int_{S_{i}}\left(-D \nabla c_{i}^{(j)}\right.$. $\mathbf{n})\left.\right|_{S_{i}} \mathrm{~d} S_{i}$, with $\mathbf{n}$ the outwards unit normal vector on $S_{i}$.

The fields $c_{i}^{(j)}$ share similar boundary conditions: zero concentration at infinity, a constant concentration $c_{0}$ prescribed at the nanobubble surface $S_{i}$, and zero flux at the solid boundary. Popov $^{\sqrt[30]{30}}$ gave the general exact solution of such a problem, using toroidal coordinates (Fig. 2). In particular, we can directly quote the mass flux (A8) calculated by Popov: 30

$$
\dot{M}_{i}=-\frac{\pi}{2} L_{i} D c_{0} f\left(\theta_{i}\right)
$$

where $D$ is the diffusion coefficient, and:

$$
f(\theta)=\frac{\sin \theta}{1+\cos \theta}+4 \int_{0}^{\infty} \frac{1+\cosh 2 \theta \tau}{\sinh 2 \pi \tau} \tanh [(\pi-\theta) \tau] \mathrm{d} \tau
$$

The first application of this result is to the problems (0): there, substituting the expression (7) for $c_{0}$ in $(9)$, we recover the result for a single nanobubble:13

$$
\dot{M}_{i}^{(0)}=-\frac{\pi}{2} L_{i} D\left[c_{s}\left(1+\frac{4 \sigma \sin \theta_{i}}{L_{i} P_{0}}\right)-c_{\infty}\right] f\left(\theta_{i}\right)
$$

To compute $\left.c_{i}^{(0)}\right|_{d}$, we need the asymptotic expression of the concentration field at order 


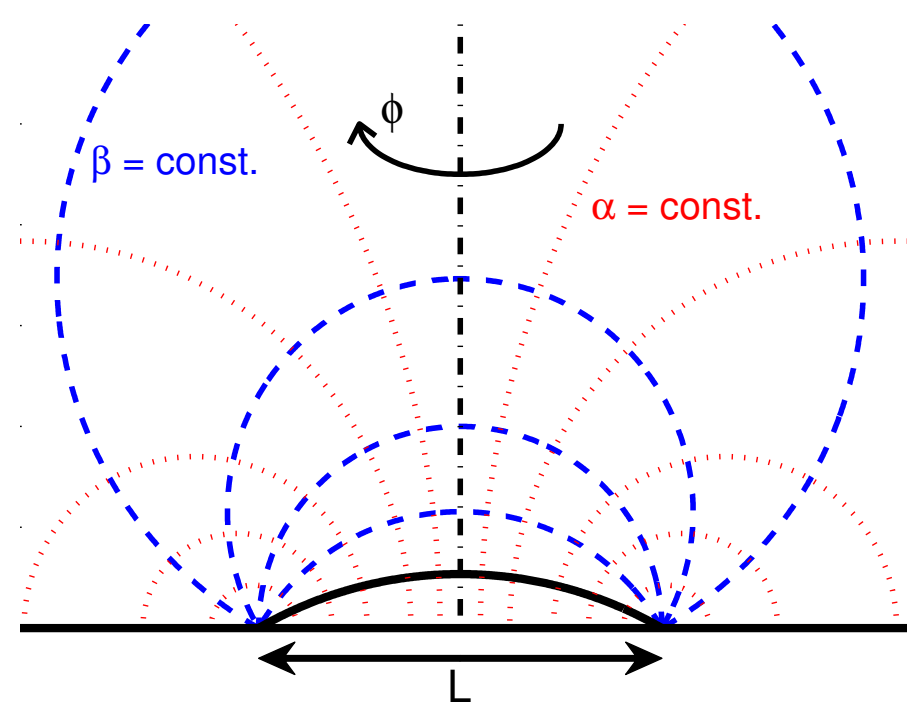

Figure 2: Sketch of the bipolar coordinates $(\alpha, \beta)$ from which the toroidal coordinates $(\alpha, \beta, \phi)$ are obtained by rotation about the vertical symmetry axis (dashed-dotted line). The bipolar coordinates are obtained from two points called foci, separated by a distance $L$. The curves at constant $\beta$ are the circles passing through the two foci (dashed curves). They interest the axis joining the foci with an angle $\beta$. Hence, this axis can be mapped to the wall, and the nanobubble of contact angle $\theta$ can be identified with the curve $\beta=\theta[2 \pi]$ (plain curve). The curves at constant $\alpha$ are the circles perpendicular to the family of circles at constant $\beta$ (dotted curves). The coordinate $\beta$ is defined on any interval of length $2 \pi$, and the coordinate $\alpha$ is defined between 0 and $+\infty$; as $\alpha \rightarrow+\infty$, the circles at constant $\alpha$ tend towards the foci, and as $\alpha \rightarrow 0^{+}$, their radius tend towards $\infty$. 
zero at large distance from the nanobubbles. We use the exact concentration field (A3) given by Popov: $\stackrel{30}{[}$

$$
\begin{aligned}
c_{i}^{(0)}\left(\alpha_{i}, \beta_{i}\right)=\left[c_{s}\right. & \left.\left(1+\frac{4 \sigma \sin \theta_{i}}{L_{i} P_{0}}\right)-c_{\infty}\right] \sqrt{2\left(\cosh \alpha_{i}-\cos \beta_{i}\right)} \\
& \times \int_{0}^{\infty} \frac{\cosh \theta_{i} \tau \cosh \left(2 \pi-\beta_{i}\right) \tau}{\cosh \pi \tau \cosh (\pi-\theta) \tau} P_{-1 / 2+\mathrm{i} \tau}\left(\cosh \alpha_{i}\right) \mathrm{d} \tau
\end{aligned}
$$

where $c_{0}$ takes the value given by Eq. (7), and where the toroidal coordinates $\left(\alpha_{i}, \beta_{i}\right)$ are defined with respect to nanobubble $i$. Despite its complex index $-1 / 2+\mathrm{i} \tau$, the Legendre function $P_{-1 / 2+\mathrm{i} \tau}$ is real valued. ${ }^{31}$ In this solution, $\beta_{i}$ is assumed to vary between $\pi-\theta_{i}$, which defines $S_{i}$, and $3 \pi-\theta_{i}$. To compute the far-field asymptotic behavior of (11), we notice that the far field corresponds to $\alpha_{i} \rightarrow 0, \cos \beta_{i} \rightarrow 1$, hence $\beta_{i}=2 \pi$. From the property of the Legendre function $P_{\nu}(1)=1$, 31 11 has the following far-field behavior:

$$
c_{i}^{(0)} \sim\left[c_{s}\left(1+\frac{4 \sigma \sin \theta_{i}}{L_{i} P_{0}}\right)-c_{\infty}\right] \frac{L_{i}}{r} \varphi\left(\theta_{i}\right)
$$

where $r$ is the distance from the center of the footprint, and $\varphi$ is defined as:

$$
\varphi(\theta)=\int_{0}^{\infty} \frac{\cosh \theta \tau}{\cosh \pi \tau \cosh (\pi-\theta) \tau} \mathrm{d} \tau
$$

A plot of this auxiliary function (where the integral must be performed numerically) is shown in Fig. 3. It is an increasing function of the contact angle, which diverges as $\theta \rightarrow \pi$. By direct integration, we have the two useful exact values: $\varphi(0)=1 / \pi$, and $\varphi(\pi / 2)=1 / 2$. The latter value enables us to cross check our calculations with the well-known solution of the Laplace equation around a sphere of radius $R: c(r)=c_{0} R / r$, with $r$ the distance to the center of the sphere. Finally, $(12)$ yields the following value:

$$
\left.c_{i}^{(0)}\right|_{d}=\left[c_{s}\left(1+\frac{4 \sigma \sin \theta_{i}}{L_{i} P_{0}}\right)-c_{\infty}\right] \frac{L_{i}}{d} \varphi\left(\theta_{i}\right)=\left[c_{s}\left(1+\frac{4 \sigma \sin \theta_{i}}{L_{i} P_{0}}\right)-c_{\infty}\right] \varepsilon \frac{L_{i}}{L_{1}} \varphi\left(\theta_{i}\right),
$$

where $\left.c_{i}^{(0)}\right|_{d}$ is the concentration field induced by the presence of nanobubble $i$, evaluated at the location of the other nanobubble $3-i$. 


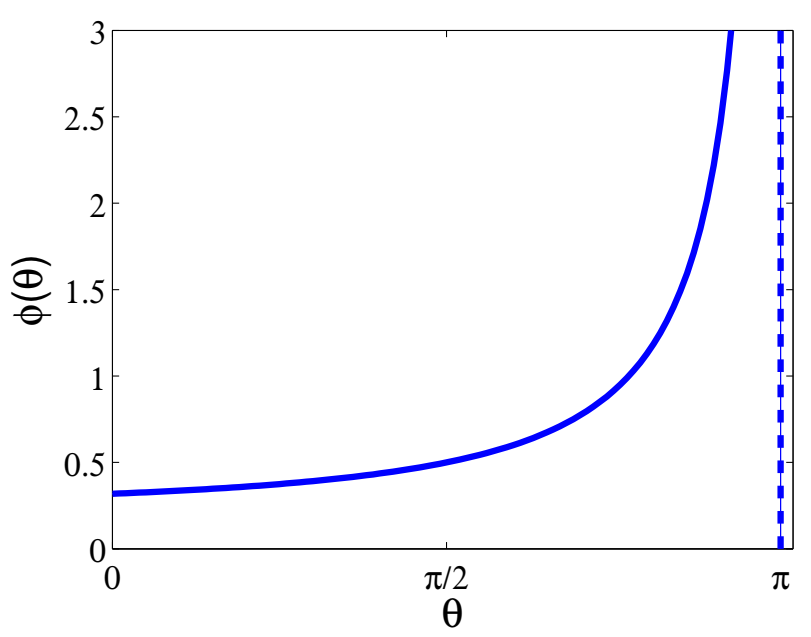

Figure 3: Plot of $\varphi(\theta)$ defined by $(13)$.

Hence, the boundary condition (8) of the problem at order 1 becomes:

$$
c_{i}^{(1)}=-\left.\varepsilon^{-1} c_{3-i}^{(0)}\right|_{d}=-\frac{L_{3-i}}{L_{1}}\left[c_{s}\left(1+\frac{4 \sigma \sin \theta_{3-i}}{L_{3-i} P_{0}}\right)-c_{\infty}\right] \varphi\left(\theta_{3-i}\right) .
$$

Substituting this value for $c_{0}$ into $(9)$, we get the first-order correction for the mass flux towards bubble $i$ :

$$
\begin{aligned}
\dot{M}_{i}^{(1)} & =-\frac{\pi}{2} L_{i} D c_{i}^{(1)} f\left(\theta_{i}\right) \\
& =\frac{\pi}{2} L_{i} D\left\{\frac{L_{3-i}}{L_{1}}\left[c_{s}\left(1+\frac{4 \sigma \sin \theta_{3-i}}{L_{3-i} P_{0}}\right)-c_{\infty}\right] \varphi\left(\theta_{3-i}\right)\right\} f\left(\theta_{i}\right) .
\end{aligned}
$$

Hence, combining (10) and (14), the mass flux towards nanobubble $i$ writes:

$$
\begin{aligned}
\dot{M}_{i}=\dot{M}_{i}^{(0)} & +\varepsilon \dot{M}_{i}^{(1)}=-\frac{\pi}{2} L_{i} \operatorname{Df}\left(\theta_{i}\right)\left\{c_{s}\left(1+\frac{4 \sigma \sin \theta_{i}}{L_{i} P_{0}}\right)-c_{\infty}\right. \\
& \left.-\varepsilon \frac{L_{3-i}}{L_{1}}\left[c_{s}\left(1+\frac{4 \sigma \sin \theta_{3-i}}{L_{3-i} P_{0}}\right)-c_{\infty}\right] \varphi\left(\theta_{3-i}\right)\right\} .
\end{aligned}
$$

Now, from the simple geometry of a spherical cap with fixed footprint diameter $L_{i}$, we get:

$$
\dot{M}_{i}=\frac{\pi}{8} \rho_{g} L_{i}^{3} \frac{\dot{\theta}_{i}}{\left(1+\cos \theta_{i}\right)^{2}}
$$

Hence, using the oversaturation parameter $\zeta$ and the length scale $L_{c}$ we get the coupled dy- 
namical system driving the evolution of the two contact angles:

$$
\left\{\begin{array}{l}
\dot{\theta}_{1}=\frac{4 D c_{s}}{\rho_{g} L_{1}^{2}}\left(1+\cos \theta_{1}\right)^{2} f\left(\theta_{1}\right)\left[\zeta-\frac{L_{c}}{L_{1}} \sin \theta_{1}-\varepsilon \frac{L_{2}}{L_{1}}\left(\zeta-\frac{L_{c}}{L_{2}} \sin \theta_{2}\right) \varphi\left(\theta_{2}\right)\right] \\
\dot{\theta}_{2}=\frac{4 D c_{s}}{\rho_{g} L_{2}^{2}}\left(1+\cos \theta_{2}\right)^{2} f\left(\theta_{2}\right)\left[\zeta-\frac{L_{c}}{L_{2}} \sin \theta_{2}-\varepsilon\left(\zeta-\frac{L_{c}}{L_{1}} \sin \theta_{1}\right) \varphi\left(\theta_{1}\right)\right]
\end{array} .\right.
$$

We stress again that this system (15) is valid up to order $\varepsilon$ included. For large nanobubble distance $\varepsilon \rightarrow 0$, the two equations decouple and reduce to the evolution equation recently derived by Lohse and Zhang:!13

$$
\dot{\theta}=\frac{4 D c_{s}}{\rho_{g} L^{2}}(1+\cos \theta)^{2} f(\theta)\left(\zeta-\frac{L_{c}}{L} \sin \theta\right)
$$

\section{Equilibrium and stability}

The system 15 is the central result of our theoretical study, as it allows us to study the equilibrium and the stability of the coupled system of two surface nanobubbles. Their equilibrium is such that $\dot{\theta}_{1}=\dot{\theta}_{2}=0$, hence:

$$
\left\{\begin{array}{l}
0=\zeta-\frac{L_{c}}{L_{1}} \sin \theta_{1}-\varepsilon \frac{L_{2}}{L_{1}}\left(\zeta-\frac{L_{c}}{L_{2}} \sin \theta_{2}\right) \varphi\left(\theta_{2}\right) \\
0=\zeta-\frac{L_{c}}{L_{2}} \sin \theta_{2}-\varepsilon\left(\zeta-\frac{L_{c}}{L_{1}} \sin \theta_{1}\right) \varphi\left(\theta_{1}\right)
\end{array}\right.
$$

Hence, the equilibrium contact angle $\theta_{i}^{(0)}$ of a single nanobubble obeys $\sin \theta_{i}^{(0)}=\zeta L_{i} / L_{c}$, in agreement with the result of ref. ${ }^{13}$ If we set: $\theta_{i}^{\text {eq }}=\theta_{i}^{(0)}+\varepsilon \theta_{i}^{(1)}+\ldots$ and insert in 17 , it immediately follows that $\theta_{i}^{(1)}=0$. Hence, the equilibrium contact angle is not modified at $\operatorname{order} \varepsilon$.

The most interesting question is whether the presence of a second nanobubble will affect the stability of the first. It was shown by Lohse \& Zhang $\frac{13}{13}$ that a pinned nanobubble was stable against dissolution, contrary to a bulk bubble, because its Laplace pressure is an increasing function of its contact angle; mathematically, $\dot{\theta}=g(\theta)$ with $\left.(\mathrm{d} g / \mathrm{d} \theta)\right|_{\theta^{\mathrm{eq}}}<0$. This stability criterion actually holds for $\theta<\pi / 2$, which corresponds to the experimental range of very small contact angles reported in the literature邽. To study the stability of the two-bubble equilibrium, we linearise the system (15) close to the equilibrium: writing $\theta_{i}=\theta_{i}^{\text {eq }}+\delta_{i}$ with $\left|\delta_{i}\right| \ll 1$, and

\footnotetext{
${ }^{1}$ For the case $\theta>\pi / 2$ stability and instability of course would be reversed.
} 
$\delta=\left(\delta_{1}, \delta_{2}\right)^{T}$, we get $\dot{\delta}=\left(4 D c_{s} / \rho_{g}\right) A \delta$ with the matrix $A$ :

$$
A=\left(\begin{array}{cc}
-\frac{L_{c}}{L_{1}^{3}}\left(1+\cos \theta_{1}^{\mathrm{eq}}\right)^{2} f\left(\theta_{1}^{\mathrm{eq}}\right) \cos \theta_{1}^{\mathrm{eq}} & \varepsilon \frac{L_{c}}{L_{1}^{3}}\left(1+\cos \theta_{1}^{\mathrm{eq}}\right)^{2} f\left(\theta_{1}^{\mathrm{eq}}\right) \varphi\left(\theta_{2}^{\mathrm{eq}}\right) \cos \theta_{2}^{\mathrm{eq}} \\
\varepsilon \frac{L_{c}}{L_{2}^{3}}\left(1+\cos \theta_{2}^{\mathrm{eq}}\right)^{2} f\left(\theta_{2}^{\mathrm{eq}}\right) \varphi\left(\theta_{1}^{\mathrm{eq}}\right) \cos \theta_{1}^{\mathrm{eq}} & -\frac{L_{c}}{L_{2}^{3}}\left(1+\cos \theta_{2}^{\mathrm{eq}}\right)^{2} f\left(\theta_{2}^{\mathrm{eq}}\right) \cos \theta_{2}^{\mathrm{eq}}
\end{array}\right)
$$

The stability of equilibrium depends on the sign of the eigenvalues of this matrix: it is stable if and only if both are negative. Now, the eigenvalues obey the polynomial equation:

$$
\begin{aligned}
0=\operatorname{det}( & A-\lambda I)=\lambda^{2}+L_{c}\left[\frac{1}{L_{1}^{3}}\left(1+\cos \theta_{1}^{\mathrm{eq}}\right)^{2} f\left(\theta_{1}^{\mathrm{eq}}\right) \cos \theta_{1}^{\mathrm{eq}}\right. \\
& \left.+\frac{1}{L_{2}^{3}}\left(1+\cos \theta_{2}^{\mathrm{eq}}\right)^{2} f\left(\theta_{2}^{\mathrm{eq}}\right) \cos \theta_{2}^{\mathrm{eq}}\right] \lambda+\frac{L_{c}^{2}}{L_{1}^{3} L_{2}^{3}}\left[1-\varepsilon^{2} \frac{L_{2}}{L_{1}} \varphi\left(\theta_{1}^{\mathrm{eq}}\right) \varphi\left(\theta_{2}^{\mathrm{eq}}\right)\right] \\
& \times\left(1+\cos \theta_{1}^{\mathrm{eq}}\right)^{2}\left(1+\cos \theta_{2}^{\mathrm{eq}}\right)^{2} f\left(\theta_{1}^{\mathrm{eq}}\right) f\left(\theta_{2}^{\mathrm{eq}}\right) \cos \theta_{1}^{\mathrm{eq}} \cos \theta_{2}^{\mathrm{eq}} .
\end{aligned}
$$

To discuss only the stability and not the growth or decay rates, we only need to discuss the sign of the coefficients of this second-order polynomial. Indeed, if we write it $\lambda^{2}+a \lambda+b, a$ equals the sum of its two roots, and $b$ their product. The assumption $\theta_{1}^{\text {eq }}<\pi / 2$ and $\theta_{2}^{\text {eq }}<\pi / 2$ guarantees that $a>0$. Hence, if $b>0$ with

$$
\begin{gathered}
b=\frac{L_{c}^{2}}{L_{1}^{3} L_{2}^{3}}\left(1+\cos \theta_{1}^{\mathrm{eq}}\right)^{2}\left(1+\cos \theta_{2}^{\mathrm{eq}}\right)^{2} f\left(\theta_{1}^{\mathrm{eq}}\right) f\left(\theta_{2}^{\mathrm{eq}}\right) \cos \theta_{1}^{\mathrm{eq}} \cos \theta_{2}^{\mathrm{eq}} \\
\times\left[1-\varepsilon^{2} \frac{L_{2}}{L_{1}} \varphi\left(\theta_{1}^{\mathrm{eq}}\right) \varphi\left(\theta_{2}^{\mathrm{eq}}\right)\right]
\end{gathered}
$$

both eigenvalues are positive and the two-nanobubble equilibrium is stable. The sign of $b$ is determined by the sign of the term $1-\varphi\left(\theta_{1}^{\text {eq }}\right) \varphi\left(\theta_{2}^{\text {eq }}\right) \varepsilon^{2} L_{2} / L_{1}$ in eq. 19]. It is positive except if $\varphi\left(\theta_{1}^{\text {eq }}\right) \varphi\left(\theta_{2}^{\text {eq }}\right) L_{1} L_{2} / d^{2}>1$. Since $\varphi(\theta)$ increases from $1 / \pi$ to $1 / 2$ when $\theta$ increases from 0 to $\pi / 2$ (Fig. 3), a necessary condition for this inequality to hold is: $L_{1} L_{2} / d^{2}>4$ or, equivalently, $\varepsilon^{2} L_{2} / L_{1}>4$, which (i) violates our assumption $\varepsilon \ll 1$, (ii) is anyway geometrically impossible, because $d>\left(L_{1}+L_{2}\right) / 2$ for two separated nanobubbles. Hence, the eigenvalues of $A$ remain positive, and the two-nanobubble equilibrium remains stable, even for different radii of curvature of the two nanobubbles. In other words: In linear order Ostwald ripening is suppressed for the neighboring surface nanobubbles. 


\section{Conclusions and outlook}

In summary, we have theoretically shown that in the framework of our analytical linear approximation, the equilibrium of a nanobubble and its stability are not affected by the presence of a second, neighboring nanobubble. This may on first sight appear puzzling when thinking in terms of Ostwald ripening, where a bigger bubble grows at the expense of smaller neighbours and which clearly holds for microbubbles in the bulk. However, once more, as in the case of a single surface nanobubbles, $\frac{13}{13}$ this bulk-bubble based intuition fails because of the pinning condition for surface nanobubbles.

The next steps to be taken are obvious: On the numerical side, just as we numerically confirmed the stability theory of ref. $\frac{13}{}$ by molecular dynamics (MD) simulations ${ }^{14}$ for a single surface nanobubbles, we will numerically confirm the present theory for the stability of a pair of diffusively interacting (pinned) surface nanobubbles. The MD simulations will also allow us to go beyond the linear approximation and explore higher order Ostwald ripening effects. On the theoretical side, the present calculation for two diffusively interacting surface nanobubbles can straightforwardly be extended to three and more surface nanobubbles. On the experimental side, the community is in urgent need of controlled and quantitative experiments for diffusively interacting surface nanobubbles. In particular, the gas oversaturation $\zeta$ should be known in these experiments, which clearly poses a challenge and may require highly controlled devices as that described in refs. $\underline{32}$

\section{Acknowledgments}

We gratefully acknowledge numerous illuminating discussions with Xuehua Zhang over the years. We also acknowledge helpful discussions with Vince Craig and Ing-Shouh Hwang and financial support from the NWO-MCEC programme and an ERC Advanced Grant.

\section{References}

(1) Lohse, D.; Zhang, X. Surface nanobubble and surface nanodroplets. Rev. Mod. Phys. 2015, $87,981-1035$. 
(2) Craig, V. S. J. Very small bubbles at surfaces - the nanobubble puzzle. Soft Matter 2011, $7,40-48$.

(3) Hampton, M. A.; Nguyen, A. V. Nanobubbles and the nanobubble bridging capillary force. Adv. Colloid Interface Sci. 2010, 154, 30-55.

(4) Attard, P. Nanobubbles: the big picture. Phys. A 2002, 314, 696-705.

(5) Attard, P. The stability of nanobubbles. Europ. Phys. J. Spec. Topics 2013, 1-22.

(6) Parker, J. L.; Claesson, P. M.; Attard, P. Bubbles, cavities and the long-ranged attraction between hydrophobic surfaces. J. Phys. Chem. 1994, 98, 8468-8480.

(7) Carambassis, A.; Jonker, L. C.; Attard, P.; Rutland, M. W. Forces Measured between Hydrophobic Surfaces due to a Submicroscopic Bridging Bubble. Phys. Rev. Lett. 1998, $80,5357-5360$.

(8) Yakubov, G. E.; Butt, H.-J.; Vinogradova, O. I. Interaction forces between hydrophobic surfaces. Attractive jump as an indication of formation of "stable" submicrocavities. J. Phys. Chem. B 2000, 104, 3407-3410.

(9) Lou, S.-T.; Ouyang, Z.-Q.; Zhang, Y.; Li, X.-J.; Hu, J.; Li, M.-Q.; Yang, F.-J. Nanobubbles on solid surface imaged by atomic force microscopy. J. Vac. Sci. Technol. B 2000, 18, $2573-2575$.

(10) Ishida, N.; Inoue, T.; Miyahara, M.; Higashitani, K. Nano bubbles on a hydrophobic surface in water observed by tapping-mode atomic force microscopy. Langmuir 2000, 16, 6377-6380.

(11) Tyrrell, J. W. G.; Attard, P. Images of nanobubbles on hydrophobic surfaces and their interactions. Phys. Rev. Lett. 2001, 87, 176104.

(12) Liu, Y.; Zhang, X. A unified mechanism for the stability of surface nanobubbles: Contact line pinning and supersaturation. J. Chem. Phys. 2014, 141, 134702.

(13) Lohse, D.; Zhang, X. Pinning and gas oversaturation imply stable single surface nanobubble. Phys. Rev. E 2015, 91, 031003(R). 
(14) Maheshwari, S.; van der Hoef, M.; Zhang, X.; Lohse, D. Stability of Surface Nanobubbles: A Molecular Dynamics Study. Langmuir 2016, 10.1021/acs.langmuir.6b00963.

(15) Zhang, X.; Chan, D. Y. C.; Wang, D.; Maeda, N. Stability of Interfacial Nanobubbles. Langmuir 2013, 29, 1017-1023.

(16) Liu, Y.; Zhang, X. Nanobubble stability induced by contact line pinning. J. Chem. Phys. 2013, 138, 014706.

(17) Liu, Y.; Wang, J.; Zhang, X.; Wang, W. Contact line pinning and the relationship between nanobubbles and substrates. J. Chem. Phys. 2014, 140, 054705.

(18) Weijs, J. H.; Lohse, D. Why Surface Nanobubbles Live for Hours. Phys. Rev. Lett. 2013, $110,054501$.

(19) Voorhees, P. W. the theory of Ostwald ripening. J. Stat. Phys. 1985, 38, 231-252.

(20) Zhang, X. H.; Maeda, N.; Craig, V. S. J. Physical properties of nanobubbles on hydrophobic surface in water and aqueous solutions. Langmuir 2006, 22, 5025-5035.

(21) Yang, S.; Dammer, S. M.; Bremond, N.; Zandvliet, H. J. W.; Kooij, E. S.; Lohse, D. Characterization of nanobubbles on hydrophobic surfaces in water. Langmuir 2007, 23, 7072-7077.

(22) Zhang, X. H.; Quinn, A.; Ducker, W. A. Nanobubbles at the interface between water and a hydrophobic solid. Langmuir 2008, 24, 4756-4764.

(23) Yang, S.; Tsai, P.; Kooij, E. S.; Prosperetti, A.; Zandvliet, H. J. W.; Lohse, D. Electrolytically generated nanobubbles on highly orientated pyrolytic graphite surfaces. Langmuir 2009, 25, 1466-1474, see also the Erratum in Langmuir 29, 5937 (2013).

(24) Zhang, X.; Uddin, M. H.; Yang, H.; Toikka, G.; Ducker, W.; Maeda, N. Effects of surfactants on the formation and the stability of interfacial nanobubbles. Langmuir 2012, 28, 10471-10477.

(25) Brotchie, A.; Zhang, X. H. Response of interfacial nanobubbles to ultrasound irradiation. Soft Matter 2011, 7, 265-269. 
(26) German, S. R.; Wu, X.; An, H.; Craig, V. S. J.; Mega, T. L.; Zhang, X. Interfacial Nanobubbles Are Leaky: Permeability of the Gas/Water Interface. ACS Nano 2014, 8, 6193-6201.

(27) in Chief: David R. Lide, E. Handbook of Chemistry and Physics, 86th ed.; Taylor and Francis, 2005.

(28) Lamb, H. Hydrodynamics; Cambridge University Press: Cambridge, 1932.

(29) A. A. Doinikov, S. T. Z. On the mutual interaction of two gas bubbles in a sound field. Phys. Fluids 1995, 7, 1923-1930.

(30) Popov, Y. O. Evaporative deposition patterns: Spatial dimensions of the deposit. Phys. Rev. E 2005, 71, 036313.

(31) Lebedev, N. N. Special Functions and their Applications; Prentice Hall: Upper Saddle River, New Jersey, 1965.

(32) Enriquez, O. R.; Hummelink, C.; Bruggert, G.-W.; Lohse, D.; Prosperetti, A.; van der Meer, D.; Sun, C. Growing bubbles in a slightly oversaturated liquid solution. Rev. Sci. Instr. 2013, 84, 065111. 